\title{
Adaptive Sensor Activity Control in Many-to-One Sensor Networks
}

\author{
Zhifeng Hu, Junshan Zhang, Senior Member, IEEE, and Lang Tong, Fellow, IEEE
}

\begin{abstract}
In this paper, we consider a many-to-one sensor network where a large number of sensors are deployed to monitor a physical environment. We explore sensor activity management to maximize the network lifetime, while meeting the quality-of-service $(\mathrm{QoS})$ requirement. Specifically, in each round the sink estimates the number of active sensors and the control information is fed back to the sensors for activity control. We start with a basic case where the total number of sensors $N$ is known, and the estimator of the number of active sensors $\hat{n}_{t}$ is accurate. We devise a sensor activity control scheme under which the number of active sensors would converge to the minimum that can meet the QoS requirement. Next, we generalize the study to the following two more complicated cases: (1) The case with known $N$ and inaccurate $\hat{n}_{t}$ : For this case, we propose a stochastic approximation algorithm to minimize the average number of active sensors while meeting the QoS requirement. (2) The case with unknown $N$ and accurate $\hat{n}_{t}$ : For this case, we cast the problem as the adaptive control of a Markov chain with unknown parameters and propose a composite optimization-oriented approach for the corresponding sensor activity control. We show that using this composite optimization-oriented approach the number of active sensors would converge to the minimum that can meet the $Q 0 S$ requirement.
\end{abstract}

Index Terms-Composite optimization, Markov chain, sensor network management, stochasticapproximation.

\section{INTRODUCTION}

$\mathbf{C}$ ONSIDER a many-to-one sensor network, in which a large number of sensors are distributed over an area to monitor a physical environment or to detect chemical and biological warfare agents. The data from the sensors are collected at the sink and used to reconstruct the original physical phenomenon [1]-[3]. The quality-of-service (QoS) of such a network is often defined as the requirement on the distortion between the original physical phenomenon and the reconstructed version, and is intimately related to the network resolution [4]. Clearly, the more active sensors used to monitor the environment, the better the QoS would potentially be. However, since the energy reserve at individual sensors is limited, using more active sensors would lead to a shortened network lifetime. Therefore, it is critical to have the right number of active sensors to achieve a good balance between the QoS and the network lifetime. Thus motivated, we devise sensor activity management schemes to control

Manuscript received September 1, 2005; revised April 20, 2006. This work was supported in part by the Office of Naval Research under Grant N00014-05-1-0636 and in part by the National Science Foundation under CAREER Award ANI-0238550 and Award CNS-0435190.

Z. Hu and J. Zhang are with the Department of Electrical Engineering, Arizona State University, Tempe, AZ 85287 USA (e-mail: zhifeng.hu@ asu.edu; junshan.zhang@asu.edu).

L. Tong is with School of Electrical and Computer Engineering, Cornell University, Ithaca, NY 14853 USA (e-mail: ltong@ece.cornell.edu).

Digital Object Identifier 10.1109/JSAC.2006.879362 the number of active sensors and analyze their performance in sensor networks accordingly.

We consider a sensor network where there are a central sink and $N$ sensors distributed over a surveillance area. Each sensor has two states, namely, ON and OFF. A sensor is switched to $\mathrm{ON}$ to monitor the environment, make measurements, and send data to the sink. A sensor can be switched to OFF to save energy when there are more than enough sensors working in the network. We assume that there is a separate broadcast channel from the sink to the sensors, from which each sensor receives feedback information to decide its activity in the next round. Note that it is assumed that sensors in the OFF (receiving) state can listen to the broadcast channel, and the power consumption in the receiving state is considerably smaller than in the ON state. The broadcast channel is assumed to be error-free, which is reasonable since the sink often has a large power supply (this is a common assumption in the literature).

As in [4], the QoS is defined as the distortion between the original physical phenomenon and the reconstructed version. In this study, we focus on homogeneous networks, which model the scenarios where the phenomenon being studiedis spatially homogeneous and the sensors are uniformly distributed. The insights obtained here will serve as a basis for studying heterogeneous networks. Under the homogeneous assumption, the average distortion is a function of the number of active sensors, and therefore, the number of active sensors becomes critical in meeting the QoS requirement. In order to minimize the network energy consumption while satisfying the QoS requirement, a target number of active sensors can be characterized as the minimum one satisfying the QoS requirement. Then, the number of active sensors can be engineered to approach the target number through the broadcast channel by the sink. For convenience, let $n_{t}$ denote the number of active sensors in round $t$, and let $D\left(n_{t}\right)$ denote the corresponding average distortion. Throughout, we assume that the sink has the knowledge of $D\left(n_{t}\right)$ and can compute the target number of active sensors accordingly. In a nutshell, the QoS requirement is given as

$$
D\left(n_{t}\right) \leq D_{o}
$$

In each round, the sink estimates the number of active sensors, denoted as $\hat{n}_{t}$. Then, the control information is broadcast to all sensors, and sensor activity management is carried out distributedly to meet the requirement in (1), while minimizing the number of active sensors.

We start with a basic case where $N$ is known and the estimator $\hat{n}_{t}$ is accurate. The basic idea of dynamic sensor activity control here can be outlined as follows. Let $n_{o p}$ be the target number of 
active sensors for a given QoS requirement. At the beginning of each round, the number of active sensors in the network is estimated, denoted as $\hat{n}_{t}$. If $\hat{n}_{t} \leq n_{o p}$, the sink computes a probability $p_{1}$ with $p_{1}=\left(n_{o p}-\hat{n}_{t}\right) /\left(N-\hat{n}_{t}\right)$ and broadcasts it. A dormant sensor would then switch to $\mathrm{ON}$ with probability $p_{1}$ in the next round. In contrast, if $\hat{n}_{t}>n_{o p}$, the sink computes $p_{2}$ with $p_{2}=\left(\hat{n}_{t}-n_{o p}\right) / \hat{n}_{t}$, and an active sensor would switch to OFF accordingly. We show that when the above activity control scheme is applied for a given $n_{o p}$, the sequence $\left\{n_{t}\right\}$ converges to $n_{o p}$ with probability one. As a result, it is desirable to set $n_{o p}$ to be $n_{a}^{*}$, where $n_{a}^{*}$ is the minimum $n_{t}$ satisfying $D\left(n_{t}\right) \leq D_{o}$.

Clearly, the knowledge of $N$ and the accuracy of $\hat{n}_{t}$ is critical to the design and the performance of sensor activity control. Thus motivated, we generalize our study to the following two settings in terms of the knowledge of $N$ and $\hat{n}_{t}$.

1) The case with known $N$ and inaccurate estimator $\hat{n}_{t}$. Obtaining an accurate estimator $\hat{n}_{t}$ may incur high complexity and may not be possible in some scenarios although algorithms effective asymptotically $(N \rightarrow \infty)$ exist [5], [6]. It is therefore reasonable to assume inaccurate (but reasonably good) estimation of $n_{t}$ for some practical scenarios. In this case, when the above sensor activity control scheme is applied, the number of active sensors would not converge to $n_{o p}$ due to the inaccuracy in $\hat{n}_{t}$. Alternatively, we devise an algorithm based on a stochastic approximation approach to adjust adaptively $n_{o p}$ so that the average number of active sensors is minimized, while $D \leq D_{o}$ is satisfied.

2) The case with unknown $N$ and accurate estimator $\hat{n}_{t}$. In some systems, possible sensor deaths or replenishment may result in unexpected change in $N$, and thus make it unknown. In this case, the sensor activity control scheme proposed above would not work well. We observe that sensor activity control in this case involves the adaptive control of a constrained Markov chain in the presence of unknown parameters, to which the direct solution does not exist in many cases. In light of this difficulty, we "incorporate" the constraint into the cost function and recast the problem in the form of the adaptive control of a Markov chain that can be solved by a composite optimization-oriented approach in [7]. We show that in this case, by defining the cost as the "indication function based distance" of $n_{t}$ from $n_{a}^{*},\left\{n_{t}\right\}$ can be made to converge to $n_{a}^{*}$ with probability one, thus solving our problem.

A natural question to ask next is how to carry out sensor activity control when $N$ is unknown and $\hat{n}_{t}$ is inaccurate. In this case, the sensor activity control schemes proposed above may not work well. Alternatively, sensor activity control here can be cast either as a partially observable Markov decision process (POMDP) problem or as a hidden Markov model (HMM) problem under constraints. Future work is needed to investigate this problem.

The rest of this paper is organized as follows. In Section II, we review some related work on sensor networks. In Section III, we consider the case where $N$ is known and $\hat{n}_{t}$ is accurate, and propose a scheme for sensor activity control under which $\left\{n_{t}\right\}$ would converge to $n_{a}^{*}$ with probability one. In Section IV, we investigate the case where $N$ is known and $\hat{n}_{t}$ is inaccurate. In this case, we use a stochastic approximation method for the adaptation of $n_{o p}$ so that the average number of active sensors is minimized in steady-state, while the QoS requirement is met. In Section $\mathrm{V}$, we study the case where $N$ is unknown and $\hat{n}_{t}$ is accurate. In this case, we model the problem as the adaptive control of a Markov chain, and propose an algorithm using a composite optimization-oriented approach under which the number of active sensors would converge to $n_{a}^{*}$ with probability one. Section VI contains the conclusion and future work.

\section{RELATED WORK}

Recent advances have made it possible to integrate monitoring and wireless communication capabilities into sensor devices. In what follows, we give a brief review for related work on many-to-one sensor networks.

Most relevant to our work is perhaps [4], in which QoS in a sensor network is investigated. Defining the QoS as the number of sensors monitoring the environment, [4] presents a sensor activity control scheme via using the Gur Game, under which the number of working sensors converges to the optimal one given in the QoS requirement. The approach in [4], however, requires that the sink successfully receives the transmissions from all the active sensors. Moreover, each sensor, regardless active or dormant, is required to keep running an algorithm to record its status and decide its activity. As a result, the complexity is relatively high. Another related work is [8], in which a price-based rate control mechanism for random access networks is devised and analyzed. Channel feedback information is used to control the aggregate packet arrival rate. It is shown that the system is stable under the control mechanism and can be stabilized at a desired operating point by choosing the parameters of the rate control scheme a priori.

The QoS provided by a sensor network is limited by the capacity of the network to make measurements of the physical environment and to transport the observed data. There has been much work along this direction. In [1], the problem of reachback communication in wireless sensor networks is considered based on network information theory, and some information theoretic bounds on the performance are presented. In [9], the communication from a cooperative sensor network to a mobile access point is considered. Three system configurations are discussed based on whether a polling channel is implemented and whether an energy limit is imposed on individual sensors. The capacity of these system configurations is derived. The work in [10] characterizes the amount of data required to sample, quantize, and encode a field densely deployed with wireless sensors, and the transport capacity of a many-to-one wireless sensor network is also given. In [11], the authors investigate the bounds for the number of sensors required to achieve a desired level of sensing accuracy. Using a random coding argument, they prove a lower bound on the "sensing capacity" of a sensor network, which characterizes the ability of a sensor network to distinguish among all states of nature.

Energy efficiency is another key factor in determining the sensor network performance. Much work has been done to enhance the energy efficiency in sensor networks. In [12], the energy consumption in many-to-one data-gathering sensor networks is analyzed, and both flat and clustering network structures are considered. In [13], the authors present a 
fluid-flow-based computational framework to study the expected lifetime and the network information capacity of a data-gathering wireless sensor network. A QoS specific information retrieval protocol is proposed in [14] to optimize the network performance under the metric of information rate per Joule. The proposed protocol enhances energy efficiency by eliminating redundant transmissions and fully exploiting the channel reception capability in a fading environment. In [15], the authors consider the problem of correlated data gathering by a network with a sink node and a tree-based communication structure, where the goal is to minimize the total transmission cost (e.g., the energy consumption). After showing the problem to be NP-hard, the authors proposed approximation algorithms for the transmission structure which yield solutions close to the optimal.

\section{The Case With KnOwn $N$ AND ACCURATE ESTIMATOR $\hat{n}_{t}$}

In this section, we assume that $N$ is known and that $\hat{n}_{t}$ is accurate. Note that this basic case is relevant to some practical scenarios. For example, in [4], it is assumed that the sink successfully receives transmissions from all the active sensors, thereby the number of active sensors is known. We note that in this case, once $n_{t}$ is estimated at the sink for a given $n_{o p}$, the probabilities $p_{1}$ and $p_{2}$ are computed as

$$
\begin{aligned}
& p_{1}=\frac{n_{o p}-\hat{n}_{t}}{N-\hat{n}_{t}} \mathcal{I}\left(\hat{n}_{t} \leq n_{o p}\right) \\
& p_{2}=\frac{\hat{n}_{t}-n_{o p}}{\hat{n}_{t}} \mathcal{I}\left(\hat{n}_{t}>_{o p}\right) .
\end{aligned}
$$

The sensors switch to ON/OFF accordingly, as noted before. The above feedback control scheme is locally optimal in each round in the sense that in computing $p_{1}$ and $p_{2}$, it maximizes the probability of transmitting to the desired state $n_{t}=n_{o p}$ in the next round.

With the above procedures carried out repeatedly at each round, $\left\{n_{t}\right\}$ would evolve as a Markov chain with states $1,2, \ldots, N$, that is,

$$
n_{t+1}=n_{t}+X_{t}^{a}-X_{t}^{b}
$$

where $X_{t}^{a}$ and $X_{t}^{b}$ are Binomial random variables, and

$$
\begin{aligned}
X_{t}^{a} & \sim \operatorname{Binomial}\left(N-\hat{n}_{t}, p_{1}\right), \\
X_{t}^{b} & \sim \operatorname{Binomial}\left(\hat{n}_{t}, p_{2}\right) .
\end{aligned}
$$

Note that $p_{1}=p_{2}=0$ implies that $n_{t_{o}}=n_{o p}$ for some $t_{o}$. That is to say, $n_{t}=n_{o p}$ is the absorbing state of the Markov chain, and all the other states are transient states. As a result, $\left\{n_{t}\right\}$ would converge in steady state to $n_{o p}$ with probability one. Therefore, if we choose $n_{o p}=n_{a}^{*}$, where

$$
n_{a}^{*}=\min \left\{n_{t}: D\left(n_{t}\right) \leq D_{o}\right\}
$$

the sequence $\left\{n_{t}\right\}$ would converge to $n_{a}^{*}$ with probability one, so that the system would maintain the minimum number of active sensors, while satisfying the distortion constraint.

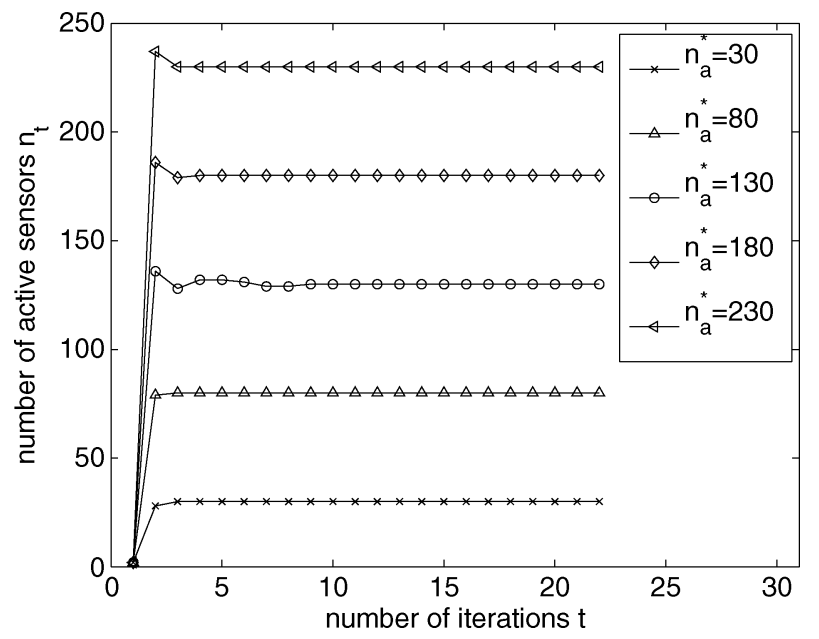

Fig. 1. Convergence of $\left\{n_{t}\right\}$ for different $n_{a}^{*}$.

Next, we characterize the absorption time, defined as the expected time it takes the system to arrive at the absorbing state. To this end, observe that the transition matrix of the Markov chain is

$$
p=\left(\begin{array}{l|l}
S & T \\
\hline 0 & 1
\end{array}\right)
$$

where the $(i j)$ th element $p_{i j}=P\left(n_{t+1}=j \mid n_{t}=i\right)$ and the index of the states are arranged such that $n_{t}=n_{o p}$ is the last one. Define $Q \triangleq(I-S)^{-1}$, where $q_{i j}$ stands for the number of times the system is expected to visit state $j$ before absorption [16]. As a result, the absorption time starting with state $i, t_{i}$, is the sum of all the entries in the $i$ th row of $Q$.

We illustrate by numerical examples the convergence of $\left\{n_{t}\right\}$ under the above feedback control scheme. In the simulation, we set $N=200$, and let $n_{a}^{*}$ take on different values. As shown in Fig. 1, it takes only a few iterations for $\left\{n_{t}\right\}$ to converge to $n_{a}^{*}$ for all cases.

\section{The CASE With KNOWN $N$ AND INACCURATE ESTIMATOR $\hat{n}_{t}$}

\section{A. Problem Formulation}

As shown in the previous section, when the estimation is accurate, the number of active sensors would converge to $n_{a}^{*}$. However, obtaining an accurate estimator $\hat{n}_{t}$ may incur high complexity and may not be possible in some systems. Alternatively, good estimation of $n_{t}$ can be achieved with low complexity estimation methods (see, e.g., [5] and [6]). In this section, we study how to carry out dynamic activity management when an inaccurate estimator $\hat{n}_{t}$ is used. Note that an ideal estimator should be dependent only on the current number of active sensors and independent of the number of active sensors at any previous time instant. This is satisfied by many estimation methods available. We assume this nature on the estimator throughout this paper.

For a given $n_{o p}$, if the scheme proposed for the first case is applied but with an inaccurate estimator $\hat{n}_{t}$ dependent only on 
$n_{t},\left\{n_{t}\right\}$ still evolves as a Markov chain. However, since $\hat{n}_{t} \neq$ $n_{t}, X_{t}^{a}$, and $X_{t}^{b}$ would not be zero even if $n_{t}=n_{o p}$. That is to say, $\left\{n_{t}\right\}$ evolves as an irreducible Markov chain and would no longer converge to $n_{o p}$. As a result, simply letting $n_{o p}=n_{a}^{*}$ as in the first case would no longer ensure the satisfaction of the QoS requirement. It is then desirable to determine a new $n_{o p}$ to ensure that the QoS requirement is satisfied in steady-state, while the average number of active sensors is minimized. Let $T\left(n_{o p}\right)$ denote the average distortion in steady-state and $\boldsymbol{E}\left[n_{t}\right]$ denote the average number of active sensors in steady-state, the problem can be put as follows:

$$
\begin{array}{ll}
\min _{n_{o p}} & E\left[n_{t}\right] \\
\text { s. t. } & T\left(n_{o p}\right) \leq D_{o} .
\end{array}
$$

\section{B. A Stochastic Approximation Approach}

Given $n_{o p}$, the distortion function $T\left(n_{o p}\right)$ depends on the stationary distribution of $\left\{n_{t}\right\}$, and hence the state-dependent transition matrices, which are difficult to characterize in general. Therefore, the exact form of $T\left(n_{o p}\right)$ is not available and (5) cannot be solved directly.

In what follows, we take a stochastic approximation approach to explore (5). For a given $n_{t}$, let $D\left(\hat{n}_{t} \mid n_{t}\right)$ denote the distortion computed from $\hat{n}_{t}$, i.e., $D\left(\hat{n}_{t} \mid n_{t}\right) \triangleq D\left(\hat{n}_{t}\right)$, where $\hat{n}_{t} \sim$ $P\left(\hat{n}_{t} \mid n_{t}\right)$. We impose the following assumption.

Condition 1: We assume that $\boldsymbol{E}_{\hat{n}_{t}}\left[D\left(\hat{n}_{t} \mid n_{t}\right)\right] \geq D\left(n_{t}\right)$.

For a given $n_{t}$, Condition 1 requires that the expectation of the distortion computed from the estimator be greater than or equal to the actual distortion. Note that this holds in many cases of practical interest, such as when $D\left(n_{t}\right)$ is a convex function and the estimator $\hat{n}_{t}$ is unbiased. Indeed, for a Gaussian sensor network, it is shown in [17] that the distortion as a function of the number of active sensors can be written as $D_{1}\left(n_{t}\right)=$ $1 /\left(a+b n_{t}\right)$. In this case, if the estimator is unbiased, we have that

$$
\begin{aligned}
\boldsymbol{E}_{\hat{n}_{t}}\left[D_{1}\left(\hat{n}_{t} \mid n_{t}\right)\right] & =\boldsymbol{E}_{\hat{n}_{t}}\left[\frac{1}{a+b \hat{n}_{t}} \mid n_{t}\right] \\
& \geq \frac{1}{a+b \boldsymbol{E}_{\hat{n}_{t}}\left[\hat{n}_{t} \mid n_{t}\right]} \\
& =\frac{1}{a+b n_{t}}
\end{aligned}
$$

since $D_{1}\left(n_{t}\right)$ is a convex function. It can be seen that Condition 1 holds in this case.

For convenience, for a given $n_{o p}$, let $P\left(n_{t} \mid n_{o p}\right)$ denote the stationary distribution of $\left\{n_{t}\right\}$ and $D\left(\hat{n}_{t} \mid n_{o p}\right)$ denote the distortion computed from the estimator in steady-state, i.e., $D\left(\hat{n}_{t} \mid n_{o p}\right) \triangleq D\left(\hat{n}_{t}\right)$, where $\hat{n}_{t} \sim \sum_{n_{t}=0}^{N} P\left(\hat{n}_{t} \mid n_{t}\right) P\left(n_{t} \mid n_{o p}\right)$. Let $\bar{D}\left(n_{o p}\right)$ denote the expectation of $D\left(\hat{n}_{t} \mid n_{o p}\right)$ with respect to $\hat{n}_{t}$. It follows that under Condition 1 :

$$
\begin{aligned}
\bar{D}\left(n_{o p}\right) & =\sum_{j=0}^{N} E_{\hat{n}_{t}}\left[D\left(\hat{n}_{t} \mid n_{t}=j\right)\right] P\left(n_{t}=j \mid n_{o p}\right) \\
& \geq \sum_{j=0}^{N} D(j) P\left(n_{t}=j \mid n_{o p}\right) .
\end{aligned}
$$

Note that $T\left(n_{o p}\right)=\sum_{j=0}^{N} D(j) P\left(n_{t}=j \mid n_{o p}\right)$. Thus, a sufficient condition to meet the constraint in (5) is that $\bar{D}\left(n_{o p}\right) \leq$ $D_{o}$. A "suboptimal" solution to (5) can be obtained by solving the following problem:

$$
\begin{array}{ll}
\min _{n_{o p}} & \boldsymbol{E}\left[n_{t}\right] \\
\text { s. t. } & \bar{D}\left(n_{o p}\right) \leq D_{o} .
\end{array}
$$

For convenience, let $n_{c}^{*}$ denote the solution to (6). Since $\bar{D}\left(n_{O p}\right)$ is monotonic decreasing and $\boldsymbol{E}\left[n_{t}\right]$ in steady-state is a monotonic increasing function of $n_{o p}, n_{c}^{*}$ would be the root of the equation $\bar{D}\left(n_{o p}\right)=D_{o}$. Observe that $D\left(\hat{n}_{t} \mid n_{o p}\right)$, whose expectation equals $\bar{D}\left(n_{o p}\right)$, can always be directly computed from $\hat{n}_{t}$. Therefore, a standard stochastic approximation approach can be used to determine $n_{c}^{*}$. Accordingly, we develop a stochastic approximation algorithm (see in Algorithm I) in what follows.

The basic idea of Algorithm I is as follows: Start with $n_{1}$ set to $n_{a}^{*}$. For each $k$, apply the control scheme defined in (2) with $n_{o p}=n_{k}^{o p}$ to the system for $i_{o}-1$ consecutive rounds. Use the estimator in the last round $\hat{n}_{k}^{i_{o}}$ to compute the distortion $D_{k}$. Compare $D_{k}$ with $D_{o}$ and use the difference to update $n_{k}^{o p}$. The objective of the algorithm is to obtain a sequence $\left\{n_{k}^{o p}\right\}$ that will converge to $n_{c}^{*}$, and therefore solve (6).

\section{Algorithm I}

1) Initialization: set $k=1$ and $i=0$; choose $n_{1}$ assuming accurate estimation.

2) If $n_{k}^{o p}>N$, let $n_{k+1}^{o p}=N$ and go to Step 5; if $n_{k}^{o p}<0$, let $n_{k+1}^{o p}=0$ and go to Step 5. Set $i=i+1$. Estimate the number of active sensors in the current round $\hat{n}_{k}^{i}$.

3) If $i=i_{o}$, go to Step 4. If $i<i_{o}$, the sink computes $p_{1}$ or $p_{2}$, with $n_{o p}=n_{k}^{o p}$ and $\hat{n}_{t}=\hat{n}_{k}^{i}$, and broadcasts it to the sensors. The sensors switch to ON/OFF accordingly. Go back to Step 2.

4) Compute the distortion from the estimator $\hat{n}_{k}^{i_{o}}$ as $D_{k}=D\left(\hat{n}_{k}^{i_{o}}\right)$. Update $n_{k}^{o p}$ as

$$
n_{k+1}^{o p}=n_{k}^{o p}+a_{k}\left(D_{k}-D_{o}\right)
$$

where $\left\{a_{k}\right\}$ is a prespecified positive decreasing sequence. 5) Set $k=k+1$ and $i=0$. Go back to Step 2 .

Note that for each $k$, if the control scheme is applied for $i_{O}+$ $l-2$ times, and the average of the estimators obtained in the last $l$ rounds is used to compute $D_{k}$, the convergence rate of $\left\{n_{k}^{o p}\right\}$ might change. Also, the choice of $i_{o}$ can affect the convergence rate. In the next section, we use numerical examples to show that generally $\left\{n_{k}^{o p}\right\}$ converges faster when $i_{o}=2$ and $l=1$.

Next, we prove that the sequence $\left\{n_{k}^{o p}\right\}$ as obtained in Algorithm I converges to $n_{c}^{*}$ in probability. Given $n_{o p}=n_{k}^{o p}$, assuming that the system has arrived at steady-state in the $i_{o}$ rounds before $D_{k}$ is computed, we have that $D_{k}=D\left(\hat{n}_{t} \mid n_{o p}=n_{k}^{o p}\right)$ and $\boldsymbol{E}_{\hat{n}_{k}^{i_{o}}}\left[D_{k}\right]=\bar{D}\left(n_{k}^{o p}\right)$. 
For convenience, define

$$
\begin{gathered}
b_{k} \triangleq-\frac{\bar{D}\left(n_{k}^{o p}\right)-D_{o}}{n_{k}^{o p}-n_{c}^{*}} \\
e_{k} \triangleq D_{k}-\bar{D}\left(n_{k}^{o p}\right) .
\end{gathered}
$$

We now have the following lemma.

Lemma 4.1: There exist constants $c_{0}, c_{1}, c_{2}>0$ such that:

1) $c_{o} \leq b_{k} \leq c_{1}, \forall k \in \mathbb{N}$;

2) $\boldsymbol{E}\left[e_{k}\right]=0,\left|e_{k}\right| \leq c_{2}, \forall k \in \mathbb{N}$.

The proof of Lemma 4.1 has been relegated to the appendix.

Now, we prove that the sequence $\left\{n_{k}^{o p}\right\}$ converges to $n_{c}^{*}$ in probability using Lemma 4.1 .

Theorem 4.1: There exists a sequence $\left\{a_{k}\right\}$ for Algorithm I such that when the sequence $\left\{a_{k}\right\}$ are used, $\left\{n_{k}^{o p}\right\}$ would converge to $n_{c}^{*}$ in probability.

Proof: Let $a_{k}=c_{4} / k$, with $c_{4}>\left(1 / c_{0}\right)$. From Lemma 4.1 , we have that

$$
\begin{aligned}
& a_{k} b_{k}>1 / k, \quad \forall k \in \mathbb{N} \\
& a_{k} b_{k}<1, \quad \forall k>k_{O}
\end{aligned}
$$

where $k_{O}$ is the smallest integer greater than $c_{4} c_{1}$.

Plugging $b_{k}$ and $e_{k}$ into (7) yields that

$$
n_{k+1}^{o p}-n_{c}^{*}=\left(n_{k}^{o p}-n_{c}^{*}\right)\left(1-a_{k} b_{k}\right)+e_{k} a_{k} .
$$

Letting $m_{k}=\sqrt{k}\left(n_{k}^{o p}-n_{c}^{*}\right)$, we have that

$m_{k}=\sqrt{k} m_{1} \prod_{j=1}^{k-1}\left(1-a_{j} b_{j}\right)+\sqrt{k} \sum_{i=1}^{k-1}\left(e_{i} a_{i} \prod_{j=i+1}^{k-1}\left(1-a_{j} b_{j}\right)\right)$.

For convenience, define

$$
\begin{aligned}
& u_{k} \triangleq \sqrt{k} m_{1} \prod_{j=1}^{k-1}\left(1-a_{j} b_{j}\right), \\
& v_{k} \triangleq \sqrt{k} \sum_{i=1}^{k-1}\left(e_{i} a_{i} \prod_{j=i+1}^{k-1}\left(1-a_{j} b_{j}\right)\right) .
\end{aligned}
$$

We begin with characterizing the mean value and the variance of $u_{k}$. Observe that

$$
\begin{aligned}
\left|u_{k}\right| & =\sqrt{k}\left|m_{1}\right| \prod_{j=1}^{k-1}\left|1-a_{j} b_{j}\right| \\
& =\sqrt{k}\left|m_{1}\right| \prod_{j=1}^{k_{o}}\left|1-a_{j} b_{j}\right| \prod_{j=k_{o}+1}^{k-1}\left|1-a_{j} b_{j}\right| \\
& \leq \sqrt{k}\left|m_{1}\right| \prod_{j=1}^{k_{o}}\left(1+\frac{c_{1} c_{4}}{j}\right) \prod_{j=k_{o}+1}^{k-1}\left(1-\frac{1}{j}\right) \\
& =\sqrt{k}\left|m_{1}\right| \frac{k_{o}}{k-1} \prod_{j=1}^{k_{o}}\left(1+\frac{c_{1} c_{4}}{j}\right)
\end{aligned}
$$

Therefore $\lim _{k \rightarrow \infty} \boldsymbol{E}\left[u_{k}\right]=0$.
Furthermore

$$
\begin{aligned}
\operatorname{var}\left(u_{k}\right) & =\boldsymbol{E}\left[\left(\sqrt{k} m_{1} \prod_{j=1}^{k-1}\left(1-a_{j} b_{j}\right)\right)^{2}\right] \\
& \leq\left(\sqrt{k} m_{1}\right)^{2} \prod_{j=1}^{k_{o}}\left(1+\frac{c_{1} c_{4}}{j}\right)^{2} \prod_{j=k_{o}+1}^{k-1}\left(1-\frac{1}{j}\right)^{2} \\
& =\left(\sqrt{k} m_{1}\right)^{2} \prod_{j=1}^{k_{o}}\left(1+\frac{c_{1} c_{4}}{j}\right)^{2}\left(\frac{k_{o}}{k-1}\right)^{2} \\
& =\frac{k}{(k-1)^{2}} m_{1}^{2} \prod_{j=1}^{k_{o}}\left(1+\frac{c_{1} c_{4}}{j}\right)^{2} k_{o}^{2} .
\end{aligned}
$$

Therefore, we conclude that $\lim _{k \rightarrow \infty} \operatorname{var}\left(u_{k}\right)=0$.

Next, we characterize the mean value and the variance of $v_{k}$

$$
\begin{aligned}
\boldsymbol{E}\left[v_{k}\right] & =\sqrt{k} \boldsymbol{E}\left[\sum_{i=1}^{k-1}\left(a_{i} e_{i} \prod_{j=i+1}^{k-1}\left(1-a_{j} b_{j}\right)\right)\right] . \\
& =\sqrt{k} \sum_{i=1}^{k-1} \boldsymbol{E}\left[\left(a_{i} \prod_{j=i+1}^{k-1}\left(1-a_{j} b_{j}\right)\right)\right] \boldsymbol{E}\left[e_{i}\right] .
\end{aligned}
$$

Since $\boldsymbol{E}\left[e_{i}\right]=0, \forall i \in \mathbb{N}$, we have that $\boldsymbol{E}\left[v_{k}\right]=0$.

To characterize the variance of $v_{k}$, observe that

$$
\begin{aligned}
\operatorname{var}\left(v_{k}\right) & =\boldsymbol{E}\left[\left(v_{k}\right)^{2}\right]-\boldsymbol{E}^{2}\left[v_{k}\right] \\
& =k \boldsymbol{E}\left[\left(\sum_{i=1}^{k-1} a_{i} e_{i} \prod_{j=i+1}^{k-1}\left(1-a_{j} b_{j}\right)\right)^{2}\right] .
\end{aligned}
$$

Since $e_{i}$ and $e_{j}$ are independent for $i \neq j$ and $\boldsymbol{E}\left[e_{i}\right]=0$ for any $i \in \mathbb{N}$, we have that

$$
\begin{aligned}
\operatorname{var}\left(v_{k}\right)= & k \boldsymbol{E}\left[\sum_{i=1}^{k-1} a_{i}^{2} e_{i}^{2} \prod_{j=i+1}^{k-1}\left(1-a_{j} b_{j}\right)^{2}\right] \\
= & k \boldsymbol{E}\left[\sum_{i=1}^{k_{o}-1} a_{i}^{2} e_{i}^{2} \prod_{j=i+1}^{k-1}\left(1-a_{j} b_{j}\right)^{2}\right] \\
& +k \boldsymbol{E}\left[\sum_{i=k_{o}}^{k-1} a_{i}^{2} e_{i}^{2} \prod_{j=i+1}^{k-1}\left(1-a_{j} b_{j}\right)^{2}\right] .
\end{aligned}
$$

Combining Lemma 4.1 with (8) and (9) yields that

$$
\begin{aligned}
k \boldsymbol{E} & {\left[\sum_{i=1}^{k_{o}-1} a_{i}^{2} e_{i}^{2} \prod_{j=i+1}^{k-1}\left(1-a_{j} b_{j}\right)^{2}\right] } \\
\leq & k \sum_{i=1}^{k_{o}-1}\left(\frac{c_{4} c_{2}}{i}\right)^{2} \prod_{j=i+1}^{k_{o}}\left(1+\frac{c_{4} c_{1}}{j}\right)^{2} \\
& \prod_{j=k_{o}+1}^{k-1}\left(1-\frac{1}{k}\right)^{2}
\end{aligned}
$$




$$
\begin{aligned}
& =\frac{k k_{o}^{2}}{(k-1)^{2}} \sum_{i=1}^{k_{o}-1}\left(\frac{c_{4} c_{2}}{i}\right)^{2} \prod_{j=i+1}^{k_{o}}\left(1+\frac{c_{4} c_{1}}{j}\right)^{2} \\
k E & {\left[\sum_{i=k_{o}}^{k-1} a_{i}^{2} e_{i}^{2} \prod_{j=i+1}^{k-1}\left(1-a_{j} b_{j}\right)^{2}\right] } \\
& \leq k \sum_{i=k_{o}}^{k-1}\left(\frac{c_{4} c_{2}}{i}\right)^{2} \prod_{j=i+1}^{k-1}\left(1-\frac{1}{k}\right)^{2} \\
& =\frac{k\left(k-k_{o}\right) c_{2}^{2} c_{4}^{2}}{(k-1)^{2}} .
\end{aligned}
$$

It follows that:

$$
\begin{aligned}
\lim _{k \rightarrow \infty} \operatorname{var}\left(v_{k}\right) \leq & \lim _{k \rightarrow \infty} \frac{k k_{o}^{2}}{(k-1)^{2}} \sum_{i=1}^{k_{o}-1}\left(\frac{c_{4} c_{2}}{i}\right)^{2} \\
& \times \prod_{j=i+1}^{k_{o}}\left(1+\frac{c_{4} c_{1}}{j}\right)^{2} \\
& +\lim _{k \rightarrow \infty} \frac{k\left(k-k_{o}\right) c_{2}^{2} c_{4}^{2}}{(k-1)^{2}} \\
= & c_{2}^{2} c_{4}^{2} .
\end{aligned}
$$

Since each term in $v_{k}$ contains $e_{i}$, we have that $v_{k}$ is independent of $u_{k}$. Therefore

$$
\begin{aligned}
\lim _{k \rightarrow \infty} \boldsymbol{E}\left[m_{k}\right] & =\lim _{k \rightarrow \infty} \boldsymbol{E}\left[u_{k}+v_{k}\right]=0, \\
\lim _{k \rightarrow \infty} \operatorname{var}\left(m_{k}\right) & =\lim _{k \rightarrow \infty} \operatorname{var}\left(u_{k}+v_{k}\right) \leq c_{2}^{2} c_{4}^{2} .
\end{aligned}
$$

It follows that:

$$
\begin{aligned}
\lim _{k \rightarrow \infty} E\left[n_{k}^{o p}\right] & =n_{c}^{*}, \\
\lim _{k \rightarrow \infty} k * \operatorname{var}\left(n_{k}^{o p}-n_{c}^{*}\right) & \leq c_{2}^{2} c_{4}^{2} .
\end{aligned}
$$

From Chebyshev's inequality, we conclude that

$$
\lim _{k \rightarrow \infty} P\left(\left|n_{k}^{o p}-n_{c}^{*}\right|>\epsilon\right) \leq \lim _{k \rightarrow \infty} \frac{c_{2}^{2} c_{4}^{2}}{k \epsilon^{2}}=0 \forall \epsilon>0
$$

i.e., $\left\{n_{k}^{o p}\right\}$ converges to $n_{c}^{*}$ in probability.

\section{Numerical Examples}

In this section, we illustrate by numerical examples the convergence of Algorithm I, and study the effects of different $i_{o}$ and $l$ on the rate of convergence. Throughout the simulations, we set $N=100, D\left(n_{t}\right)=1 / n_{t}$ and $\hat{n}_{t}=n_{t}+z$, where $z \sim N(0,1)$.

To verify the convergence of the sequence $\left\{n_{k}^{o p}\right\}$, we fix $i_{o}=2$ and $l=1$, and choose different pairs of $D_{o}$ and $n_{1}$. For each pair of $D_{o}$ and $n_{1}$, we let $a_{k}$ take on different values in $\{10 / k, 100 / k, 1000 / k, 3000 / k, 7000 / k\}$, and investigate the convergence behavior of $\left\{n_{k}^{o p}\right\}$. As shown in Fig. $2,\left\{n_{k}^{o p}\right\}$ converges when $a_{k}$ takes on the values in

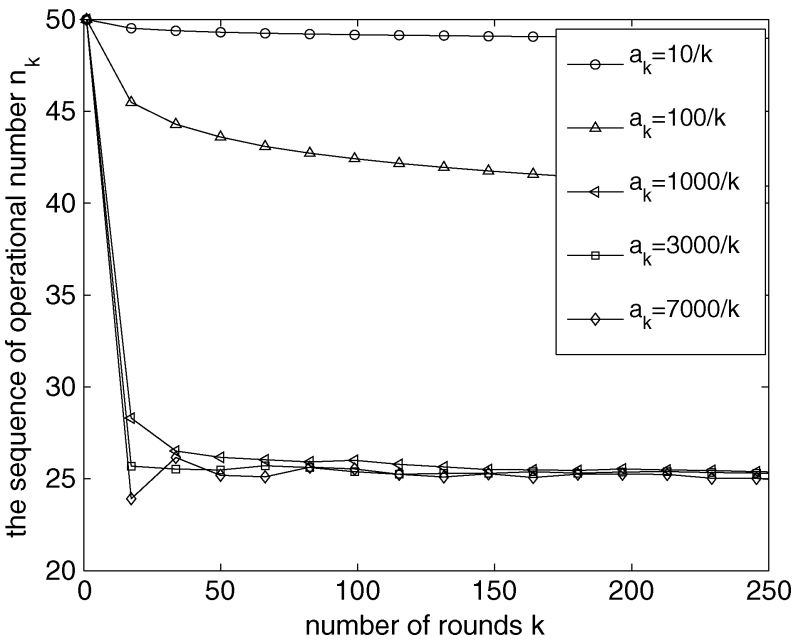

Fig. 2. Convergence performance for different $a_{k}$.

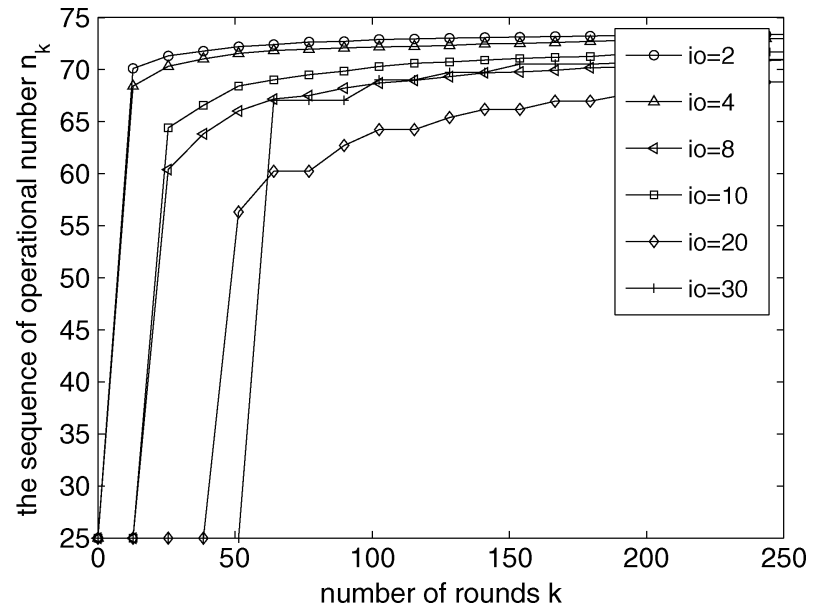

Fig. 3. Convergence performance for different $i_{o}$.

$\{1000 / k, 3000 / k, 7000 / k\}$, but may not converge when $a_{k}$ equals $10 / k$ or $100 / k$. This corroborates the fact that it is necessary to have $a_{k}>\left(1 / c_{0} k\right)$, as pointed out in the proof of Theorem 4.1.

Next, we investigate the impact of $i_{o}$ and $l$ on the convergence rate of $\left\{n_{k}^{o p}\right\}$. Intuitively, increasing $i_{o}$ and $l$ would make the time the system stays at $n_{k}^{o p}$ longer, but it is also likely to decrease the number of rounds necessary for convergence. To get a more concrete sense, we investigate the convergence rate under several numeric values of $i_{o}$ and $l$. We set $l=1$ and let $i_{o}$ take on different values in $\{2,4,8,10,20,30\}$. To compare the convergence rates on the same time scale, $\left(i_{o}+l\right) k$ is used for the horizontal axis instead of $k$. It can be seen from Fig. 3 that the fastest convergence rate is achieved when $i_{o}$ equals 2 . This indicates that the increment in the time length of every round outweighs the decrement in the number of rounds necessary for convergence when $i_{o}$ is increased. Finally, in Fig. 4, we set $i_{o}=2$ and investigate the impact of $l$ on the convergence rate. The value of $l$ is chosen from $\{1,2,4,8,10,20\}$. It can be seen that the fastest convergence rate occurs when $l=1$. 


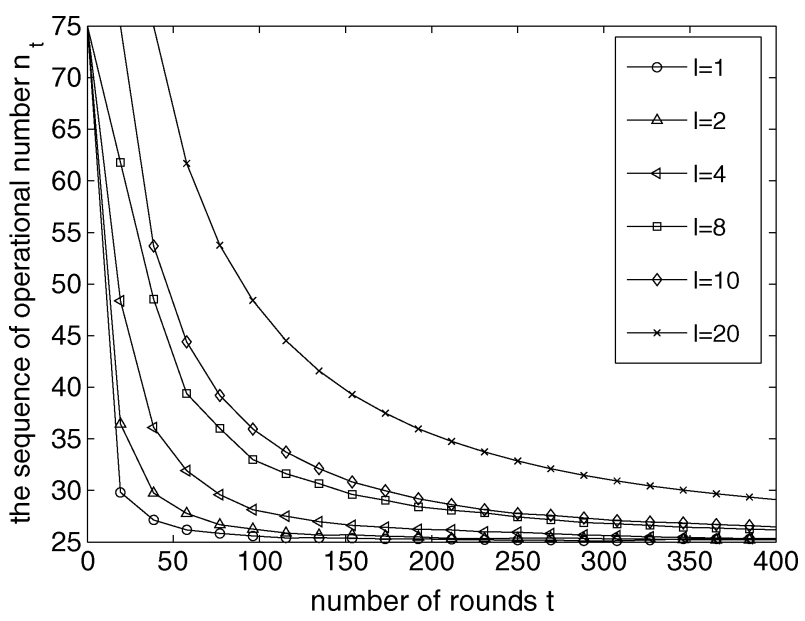

Fig. 4. Convergence performance for different $l$.

\section{The Case With UnKNown $N$ AND ACCURATE ESTIMATOR $\hat{n}_{t}$}

\section{A. Problem Formulation}

In the previous two sections, $N$ is assumed to be known at the sink. In some practical scenarios, however, the total number of sensors may change due to sensor deaths or replenishment, or simply because some sensors are in the sleep mode and cannot listen. As a result, $N$ would be unknown. Next, we study how to carry out sensor activity control in these scenarios. Note that the estimation of $n_{t}$ can be based on the observations from the active sensors, whereas the estimation of $N$ is more challenging due to the fact that there are always some dormant sensors. In what follows, we assume that $\hat{n}_{t}$ is accurate for tractability.

Since $N$ is unknown, the sensor activity control scheme used in the previous cases may not work well here, and a new scheme is needed to address the needs of parameter estimation and activity control simultaneously. To this end, define a stationary policy as a mapping $\mu(\cdot): \hat{X} \rightarrow A$, where $\hat{X}$ is the range of $\hat{n}_{t}$ and $A$ is the action space defined as $A \triangleq\left\{a=\left(p_{1}, p_{2}\right) \mid 0 \leq\right.$ $\left.p_{1} \leq 1,0 \leq p_{2} \leq 1\right\}$. Under a given policy, $\left\{n_{t}\right\}$ evolves as in (3). Let $\Xi$ denote the set of all stationary policies. The problem boils down to finding the optimal policy in $\Xi$ under which the number of active sensors would be minimized, while the distortion constraint is satisfied, with $N$ unknown. Observe that this problem points to the adaptive control of a constrained Markov chain in the presence of unknown parameters, the direct solution to which does not exist in many cases. In light of this difficulty, we "incorporate" the constraint into the cost function and recast the problem in the form of the adaptive control of a Markov chain that can be solved by a composite optimization-oriented approach in [7]. Roughly speaking, this composite optimization-oriented approach generates a sequence of actions that minimize the cost functional of the Markov chain control problem. To make it applicable to our problem, it is critical to properly define the cost functional. To this end, we choose a certain form for the cost functional and prove that in this case, the solution to the Markov chain control problem solves our problem, i.e., the average number of active sensors is minimized, while the distortion constraint is satisfied.

\section{B. Composite Optimization-Oriented Approach}

\section{Algorithm II}

Choose a function $g(t)$ such that $g(t)>0, g(t) \rightarrow 0$ and

$\operatorname{tg}(t) \rightarrow \infty$ as $t \rightarrow \infty$.

For $t=1,2, \ldots$, follow Steps 1-3.

1) Find the set

$$
\begin{aligned}
H_{t}=\left\{N_{o} \in H: v_{t}\left(a^{t-1},\right.\right. & \left.N_{o}\right) \\
& \left.\leq v_{t}\left(a^{t-1}, N_{o}^{\prime}\right)+g(t), \forall N_{o}^{\prime} \in H\right\} .
\end{aligned}
$$

2) Compute

$$
\left(\mu_{t}^{*}(\cdot), N_{t}^{*}\right)=\underset{\mu(\cdot) \in \Xi, N_{o} \in H_{t}}{\arg \min } J\left(\mu(\cdot), N_{o}\right) .
$$

3) Estimate $n_{t}$. Apply $a_{t}=\mu_{t}^{*}\left(\hat{n}_{t}\right)$ and observe the transition $n_{t} \rightarrow n_{t+1}$. Update $v_{t}\left(a^{t-1}, N_{o}\right) \rightarrow v_{t+1}\left(a^{t}, N_{o}\right)$ for all $N_{o} \in H$. Let $t=t+1$ and go back to Step 1 .

For the Markov chain of $\left\{n_{t}\right\}$, let $c(i, j, a)$ denote the cost incurred at time $t$ when action $a$ is applied, $n_{t}=i$ and $n_{t+1}=j$. We assume that $N \in H \triangleq\left\{1,2, \ldots, N_{u}\right\}$, where $N_{u}$ denotes an upper bound on $N$. For any $N_{o} \in H$, let $J\left(\mu(\cdot), N_{o}\right)$ denote the long run average cost $\lim _{t \rightarrow \infty}(1 / t) \sum_{s=1}^{t} c\left(n_{s}, n_{s+1}, a_{s}\right)$ when policy $\mu(\cdot)$ is applied to the system and $N=N_{o}$. The adaptive control of a Markov chain with unknown parameters can be formulated as follows:

$$
\mu^{*}(\cdot)=\underset{\mu(\cdot) \in \Xi}{\arg \min } J(\mu(\cdot), N)
$$

where $N$ is unknown.

To solve (12), a common procedure calls for an on-line estimation of the unknown parameter and the minimization of the cost functional using the estimate in lieu of the true parameter. It is well known that this "certainty equivalence" (CE) solution may fail to achieve the optimal performance, even asymptotically [7]. Thus motivated, we use a composite optimization-oriented approach developed in [7]. Simply put, this approach defines a new cost functional that addresses the estimation and control aspects simultaneously. The new cost functional can yield an algorithm that generates a sequence of actions that minimizes the original cost functional almost surely.

Let $a^{t}$ denote the action history, i.e., $a^{t}=\left(a_{1}, a_{2}, \ldots, a_{t}\right)$, and $\forall N_{o} \in H$, define

$$
\begin{aligned}
v_{t}\left(a^{t-1}, N_{o}\right) \triangleq & \frac{1}{t} \sum_{s=1}^{t-1} \sum_{j=1}^{N_{u}} p\left(n_{s}, j ; a_{s}, N_{o}\right) c\left(n_{s}, j, a_{s}\right) \\
& -\frac{1}{t} \sum_{s=1}^{t-1} c\left(n_{s}, n_{s+1}, a_{s}\right) \log p\left(n_{s}, n_{s+1} ; a_{s}, N_{o}\right)
\end{aligned}
$$

where $p\left(i, j ; a, N_{o}\right)$ denotes the state transition probability from $n_{t}=i$ to $n_{t}=j$ assuming that action $a$ is taken and $N=N_{o}$. 
A general algorithm based on $v_{t}\left(a^{t-1}, N_{o}\right)$ has been developed in [7] to characterize the solution to (12) (see Algorithm II).

Note that the change of $N$ occurs on a much larger time scale than that of $n_{t}$. Therefore, we assume that $N$ does not change in the duration of Algorithm II. Under mild conditions on $J\left(\mu(\cdot), N_{o}\right)$, the convergence of Algorithm II is established [7]. For convenience, we restate the result here.

Lemma 5.1: Let $\left\{a_{t}\right\}$ be a control sequence generated by the proposed adaptive algorithm and $\mu^{*}(\cdot)$ be the policy that minimizes $J(\mu(\cdot), N)$. It follows that:

$$
\lim _{t \rightarrow \infty} \frac{1}{t} \sum_{s=1}^{t} c\left(n_{s}, n_{s+1}, a_{s}\right)=J\left(\mu^{*}(\cdot), N\right) \text { a.s. }
$$

Based on Lemma 5.1, Algorithm II generates a sequence of actions that minimizes the cost functional almost surely. To make the algorithm applicable to our problem here, it is critical to properly define the cost functional. To this end, we let $c(i, j, a) \triangleq \mathcal{I}\left(j \neq n_{a}^{*}\right)$ and $J\left(\mu(\cdot), N_{o}\right) \triangleq \lim _{t \rightarrow \infty}(1 / t) \sum_{s=1}^{t} \mathcal{I}\left(n_{s} \neq n_{a}^{*}\right)$ when policy $\mu(\cdot)$ is applied and the total number of sensors equals $N_{o}$.

Note that in the problem under consideration, for any $i \in \hat{X}$, $a \in A, N_{o}, N_{o}^{\prime} \in H$ with $N_{o} \neq N_{o}^{\prime}$, we have that

$$
\begin{aligned}
{\left[p\left(i, 1 ; a, N_{o}\right), \ldots p\left(i, N_{u} ; a, N_{o}\right)\right] } & \\
& \neq\left[p\left(i, 1 ; a, N_{o}^{\prime}\right), \ldots p\left(i, N_{u} ; a, N_{o}^{\prime}\right)\right] .
\end{aligned}
$$

Hence, $g(t)$ can be set equal to 0 , leading to the achievement of the maximum possible convergence rate [7]. Given that $g(t)=$ 0, Steps 1 and 2 in Algorithm II boil down to

$$
\begin{aligned}
N_{t} & =\underset{N_{o} \in H}{\arg \min } v_{t}\left(a^{t-1}, N_{o}\right) \\
\mu_{t}^{*}(\cdot) & =\underset{\mu(\cdot) \in \Xi}{\arg \min } J\left(\mu(\cdot), N_{t}\right) .
\end{aligned}
$$

Recall from Section II that under the control scheme defined in (2) with $n_{o p}=n_{a}^{*},\left\{n_{t}\right\}$ converges to $n_{a}^{*}$ with probability one; for convenience, let $\mu_{o}(N)$ denote the scheme for a given $N$. It follows that $J\left(\mu_{o}\left(N_{o}\right), N_{o}\right)=0$ for any $N_{o} \in H$. Since $J\left(\mu(\cdot), N_{o}\right)$ is nonnegative, we conclude that $\mu_{o}\left(N_{o}\right)$ achieves the minimum value of $J\left(\mu(\cdot), N_{o}\right)$, thereby giving a solution to Step 2 in Algorithm II.

\section{Algorithm III}

For $t=1,2, \ldots$, apply Steps 1 and 2 .

1) Compute $N_{t}=\arg \min _{N_{o} \in H} v_{t}\left(a^{t-1}, N_{o}\right)$; estimate $n_{t}$; compute $p_{1}$ or $p_{2}$ using (2) with $n_{o p}=n_{a}^{*}$ and $N=N_{t}$ and broadcast it to all the sensors.

2) The sensors switch to ON/OFF using $p_{1}$ or $p_{2}$. After the transition $n_{t} \rightarrow n_{t+1}$, estimate $n_{t+1}$. Stop if $n_{t+1}=n_{a}^{*}$. Update $v_{t}\left(a^{t-1}, N_{o}\right) \rightarrow v_{t+1}\left(a^{t}, N_{o}\right)$ for all $N_{o} \in H$. Let $t=t+1$ and go back to Step 1 .
Finally, with the above definition of $c(i, j, a)$ and $J\left(\mu(\cdot), N_{o}\right), v_{t}\left(a^{t-1}, N_{o}\right)$ can be updated as follows:

$$
\begin{aligned}
v_{0}\left(N_{o}\right)= & 0 \\
v_{t+1}\left(a^{t}, N_{o}\right)= & \frac{t}{t+1} v_{t}\left(a^{t-1}, N_{o}\right) \\
& +\frac{1}{t+1}\left(1-p\left(n_{t}, n_{a}^{*} ; a_{t}, N_{o}\right)\right) \\
& -\frac{1}{t+1} \log p\left(n_{t}, n_{t+1} ; a_{t}, N_{o}\right) \\
& \times \mathcal{I}\left(n_{t+1} \neq n_{a}^{*}\right) .
\end{aligned}
$$

In summary, a simplified algorithm can be used for sensor activity control in the presence of the unknown $N$ (see Algorithm III).

Next, we prove that under Algorithm III, $\left\{n_{t}\right\}$ converges to $n_{a}^{*}$ with probability one. As a result, the average number of active sensors is minimized, while ensuring the satisfaction of the distortion constraint. This is established by the following theorem.

Theorem 5.1: When Algorithm III is applied to the system, we have that

$$
\lim _{t \rightarrow \infty} P\left(n_{t}=n_{a}^{*}\right)=1 .
$$

Proof: We prove Theorem 5.1 in two steps.

Step 1) We claim that there exists a stationary policy $\mu_{d}(\cdot) \in$ $\Xi$ such that when $\mu_{d}(\cdot)$ is applied to the system, the following equality is satisfied:

$$
J\left(\mu_{d}(\cdot), N\right)=0 .
$$

We prove this claim by construction. For any $N_{o} \in$ $H$, define $\mu_{o}\left(N_{o}\right): \hat{n}_{t} \rightarrow\left(p_{1}, p_{2}\right)$ as follows:

$$
\begin{aligned}
& p_{1}=\frac{n_{a}^{*}-\hat{n}_{t}}{N_{o}-\hat{n}_{t}} \mathcal{I}\left(\hat{n}_{t} \leq n_{a}^{*}\right) \\
& p_{2}=\frac{\hat{n}_{t}-n_{a}^{*}}{\hat{n}_{t}} \mathcal{I}\left(\hat{n}_{t}>n_{a}^{*}\right) .
\end{aligned}
$$

Clearly, $\mu_{o}\left(N_{o}\right) \in \Xi$ for any $N \leq N_{o} \leq N_{u}$. Moreover, when $N_{o}$ equals $N$, the policy $\mu_{o}\left(N_{o}\right)$ boils down to the same control scheme as used in Section II. As a result, we have that $\lim _{t \rightarrow \infty} P\left(n_{t}=\right.$ $\left.n_{a}^{*}\right)=1$ and $J\left(\mu_{o}(N), N\right)=0$ when $\mu_{o}(N)$ is applied to the system.

Step 2) We claim that with probability one there exists some round $t_{o} \in \mathbb{N}$ such that $n_{t}=n_{a}^{*}$ whenever $t \geq t_{o}$ when Algorithm III is applied to the system.

First, we show that with probability one there exists some round $t_{o} \in \mathbb{N}$ for which $n_{t_{o}}=n_{a}^{*}$ when Algorithm III is applied to the system. This follows directly from (13) and (16), because

$$
\begin{aligned}
\lim _{t \rightarrow \infty} \frac{1}{t} \sum_{s=1}^{t} \mathcal{I}\left(n_{s} \neq n_{a}^{*}\right) & =J\left(\mu^{*}(\cdot), N\right) \\
& \leq J\left(\mu_{d}(\cdot), N\right) \\
& =0 \text { a.s. }
\end{aligned}
$$




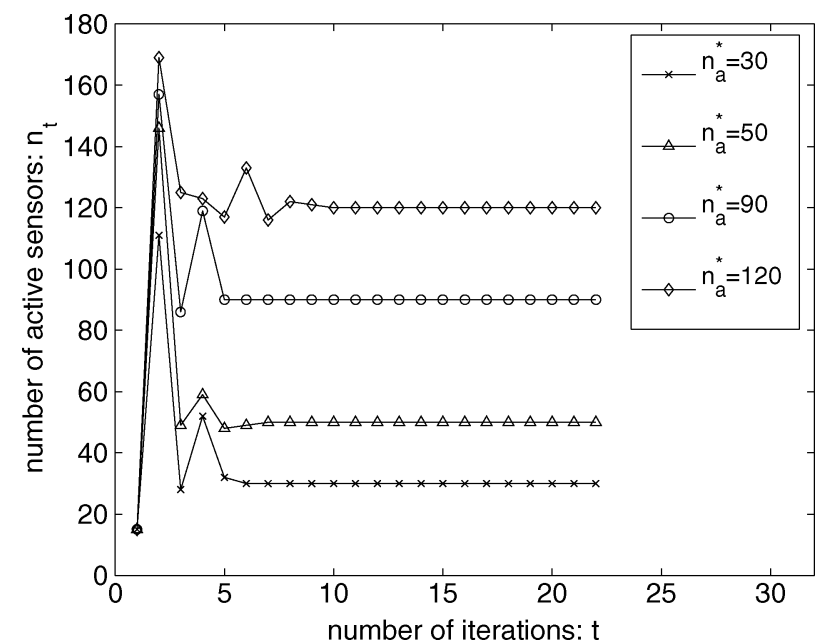

Fig. 5. Convergence of $\left\{n_{t}\right\}$ for different $n_{a}^{*}$.

It follows that $p_{1}=0$ and $p_{2}=0$ at time $t_{o}$ from Step 2 in Algorithm III. Therefore, $n_{t_{o}+1}=n_{t_{o}}=n_{a}^{*}$. By induction, for any $t \geq t_{o}, n_{t}$ equals $n_{a}^{*}$ with probability one. This finishes step 2.

We conclude that there exists some $t_{o} \in \mathbb{N}$ such that $n_{t}=n_{a}^{*}$ whenever $t \geq t_{o}$ when Algorithm III is applied to the system, thereby completing the proof.

\section{Numerical Examples}

In this section, we illustrate by numerical examples the convergence of $\left\{n_{t}\right\}$ under Algorithm III. In the simulation, $N$ is fixed but unknown to the sink. Algorithm III is applied for different values of $n_{a}^{*}$. In Fig. 5, the sequence $\left\{n_{t}\right\}$ is plotted for each given $n_{a}^{*}$. It is clear that $\left\{n_{t}\right\}$ converges to $n_{a}^{*}$ and Algorithm III works well for all cases.

\section{CONCLUSION AND FutURE WORK}

In this paper, we study sensor activity control under QoS constraints in many-to-one homogeneous sensor networks. Specifically, feedback control schemes are devised to minimize the number of active sensors, while ensuring certain QoS constraint. We start with a simple case where $N$ is known and $\hat{n}_{t}$ is accurate. In this case, a sensor activity control scheme is devised under which the number of active sensors would converge to the minimum one that can meet the QoS requirement. We then extend the study to the following two cases.

Case 1) The case with known $N$ and inaccurate estimator $\hat{n}_{t}$ : In this case, a stochastic approximation method is proposed to minimize the average number of active sensors, while meeting the QoS requirement.

Case 2) The case with unknown $N$ and accurate estimator: In this case, we model the problem as the adaptive control of a Markov chain with unknown parameters.

A composite optimization-oriented approach is proposed for the problem under which the number of active sensors would converge to the minimum one that can meet the QoS requirement.

A more challenging case that may arise is when $N$ is unknown and $\hat{n}_{t}$ is inaccurate. The sensor activity control scheme used in the first two cases may not work well for this case. Alternatively, by defining a stationary policy as a one-to-one mapping from the estimator to the action consisting of the two probabilities that are fed back to the sensors, the problem may be cast as the search of the optimal policy under which the number of active sensors is minimized, while the QoS requirement is satisfied. Depending on the modeling for the estimation inaccuracy, sensor activity control therein can be cast either as a POMDP problem or as a HMM problem under constraints. However, both techniques are quite involved, and none has been found yet that is ready to be applied to the problem here. We are currently working on this more challenging problem.

\section{APPENDIX I}

PROOF OF LEMMA 4.1

1) Since $\bar{D}\left(n_{c}^{*}\right)=D_{o}$, it follows that:

$$
b_{k}=-\frac{\bar{D}\left(n_{k}^{o p}\right)-\bar{D}\left(n_{c}^{*}\right)}{n_{k}^{o p}-n_{c}^{*}} .
$$

Since $\bar{D}\left(n_{o p}\right)$ is differentiable and strictly decreasing on $[0, N]$, it has a negative first derivative over the interval. Since $[0, N]$ is compact, the derivative over this interval has both a maximum and a minimum value. Define $c_{0} \triangleq \min _{0 \leq n_{o p} \leq N}-\left(d \bar{D}\left(n_{o p}\right) / d n_{o p}\right)$ and $c_{1} \triangleq \max _{0 \leq n_{o p} \leq N}-\left(d \bar{D}\left(n_{o p}\right) / d n_{o p}\right)$. By the Mean Value Theorem, when $n_{k}^{o p} \in[0, N]$, we have that $c_{0} \leq b_{k} \leq c_{1}$.

2) By the definition of $e_{k}$, we have $\boldsymbol{E}\left[e_{k}\right]=0$ for any $k \in \mathbb{N}$. Let $c_{2}=D(0)-D(N)$, then by the definition of $e_{k}$, we have $\left|e_{k}\right| \leq c_{2}$ for any $k \in \mathbb{N}$.

\section{REFERENCES}

[1] J. Barros and S. D. Servetto, "On the capacity of the reachback channel in wireless sensor networks," in Proc. IEEE Workshop on Multimedia Signal Processing (Special session on Signal Processing for Wireless Networks), St. Thomas, Virgin Islands, Dec. 2002, pp. 408-411.

[2] P. Venkitasubramaniam, S. Adireddy, and L. Tong, "Sensor networks with mobile access: Optimal random access and coding," IEEE J. Sel. Areas Commun (Special Issue on Sensor Networks), vol. 22, no. 6, pp. 1058-1068, 2004.

[3] L. Tong, Q. Zhao, and S. Adireddy, "Sensor networks with mobile agents," in Proc. IEEE Military Commun. Conf., Boston, MA., Oct. 2003, pp. 688-693.

[4] R. Iyer and L. Kleinrock, "QoS control for sensor networks," in Proc. IEEE Int. Conf. Commun., May 2003, pp. 517-521.

[5] C. Budianu, S. Ben-David, and L. Tong, "Estimation of number of operating sensors in large-scale sensor networks with mobile access," IEEE Trans. Signal Process., pp. 1703-1715, May 2005.

[6] A. Leshem and L. Tong, "Estimating sensor population via probabilistic sequential polling," IEEE Signal Process. Lett., vol. 12, no. 5, pp. 395-398, May 2005.

[7] R. A. Milito and J. B. Cruz, Jr., "An optimization-oriented approach to the adaptive control of Markov chains," IEEE Trans. Autom. Control, vol. 32, no. 9, pp. 754-762, 1987.

[8] C. Yuen and P. Marbach, "Price-based rate control in random access networks," IEEE/ACM Trans. Netw., to be published.

[9] Z. Yang and L. Tong, "Capacity of cooperative sensor networks with misinformed nodes," IEEE Trans. Inf. Theory, vol. 51, no. 12, pp. 4118-4133, Dec. 2005.

[10] D. Marco, E. Duarte-Melo, M. Liu, and D. L. Neuhoff, "On the many-to-one transport capacity of a dense wireless sensor network and the compressibility of its data," in Proc. Int. Workshop on Inf. Process. Sensor Netw., Palo Alto, CA, Apr. 2003, pp. 1-16. 
[11] Y. Rachlin, R. Negi, and P. Khosla, "Sensing capacity for discrete sensor network applications," in Proc. 4th Int. Symp. Inf. Process. Sensor Netw., Los Angeles, CA, Apr. 2005, pp. 126-132.

[12] E. Duarte-Melo and M. Liu, "Energy efficiency in many-to-one communications in wireless networks," in Proc. IEEE 45th Midwest Symp. Circuits Syst., Tulsa, OK, Aug. 2002, pp. 615-618.

[13] E. Duarte-Melo, M. Liu, and A. Misra, "An efficient and robust computational framework for studying lifetime and information capacity in sensor networks," ACM Mobile Networks and Applications (MONET) (Special Issue on Energy Constraints and Lifetime Performance in Wireless Sensor Networks), pp. 811-824, 2004.

[14] Q. Zhao and L. Tong, "Energy-efficient information retrieval for correlated source reconstruction in sensor networks," IEEE Trans. Wireless Commun., 2007, to be published.

[15] R. Cristescu, B. Beferull-Lozano, M. Vetterli, and R. Wattenhofer, "On network correlated data gathering with explicit communication: Np-completeness and algorithms," IEEE/ACM Trans. Netw., pp. 41-54, 2006.

[16] Isaacson and Madsen, Markov Chains Theory and Applications, 1st ed. New York: Wiley, 1976

[17] M. Gastpar and M. Vetterli, "Power, spatio-temporal bandwidth, and distortion in large sensor networks," IEEE J. Sel. Areas Commun., vol. 23, no. 4, pp. 745-754, Apr. 2005.

Zhifeng Hu received the B.S. degree in electrical engineering from Shanghai Jiaotong University, Shanghai, China, in 2003 and the M.S. degree in electrical engineering from Arizona State University, Tempe, in 2005. He is now working towards the Ph.D. degree at Arizona State University.

His main interested areas include stochastic control and optimization in wireless sensor networks.

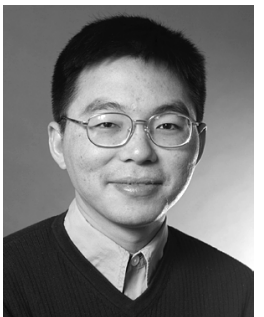

Junshan Zhang (S'98-M'00-SM'06) received the Ph.D. degree from the School of Electrical and Computer Engineering, Purdue University, West Lafayette, IN, in 2000.

He joined the Department of Electrical Engineering, Arizona State University, Tempe, in August 2000, where he is currently an Associate Professor. His research interests fall in the general area of wireless networks, spanning from the networking layer to the physical layer. His current research focuses on fundamental problems in wireless ad hoc networks and sensor networks, including cross-layer optimization and design, network management, network information theory, and stochastic analysis.

Dr. Zhang was a recipient of the Office of Naval Research (ONR) Young Investigator Award in 2005 and the National Science Foundation (NSF) CAREER Award in 2003. He has also received the Outstanding Research Award from the IEEE Phoenix Section in 2003. He was Chair of the IEEE Communications and Signal Processing Phoenix Chapter from January 2001 to December 2003. He has served as a TPC Co-Chair for IPCCC 2006 and TPC Vice-Chair for ICCCN 2006, and will be the General Chair for the IEEE Communication Theory Workshop 2007. He has been on the technical program committees for many conferences, including INFOCOM, SECON, GLOBECOM, ICC, MOBIHOC, BTOADNETS, and SPIE ITCOM. He has been an Associate Editor for the IEEE TRANSACTIONS ON WIRELESS COMMUNICATIONS since 2004.

Lang Tong (M'91-SM'01-F'05) received the B.E. degree from Tsinghua University, Beijing, China, in 1985 and the Ph.D. degree in electrical engineering from the University of Notre Dame, Notre Dame, IN, in 1991.

He joined Cornell University, Ithaca, NY, in 1998, where he is now a Professor. Prior to joining Cornell University, he was on the faculty at West Virginia University and the University of Connecticut. He was also the 2001 Cor Wit Visiting Professor at the Delft University of Technology. He was a Postdoctoral Research Affiliate at the Information Systems Laboratory, Stanford University, in 1991.

Dr. Tong received the Outstanding Young Author Award from the IEEE Circuits and Systems Society, the 2004 Best Paper Award (with Min Dong) from the IEEE Signal Processing Society, and the 2004 Leonard G. Abraham Prize Paper Award from the IEEE Communications Society (with P. Venkitasubramaniam and S. Adireddy). He also received the Young Investigator Award from the Office of Naval Research. 